\title{
Interaction and Interconnection Between 802.16e \& 802.11s
}

\author{
Tarek Bchini and Mina Ouabiba \\ ARTIMIA, Malakoff \\ France
}

\section{Introduction}

\subsection{Problematic}

With the rapid evolution of wireless and mobile networks, and the emergence of several standards that use different technologies, the problem of compatibility between these technologies, or the transition from one technology to the other by the mobile station without interruption of services, becomes a real challenge to face, to ensure a good Quality of Service (QoS) for the client.

In this context, we will analyze Mobile Stream Control Transport Protocol (MSCTP) and IEEE 802.21 technology as two vertical handover mechanisms between two of mobile networks: IEEE 802.11s, and mobile WIMAX. The simulations will be run under Network Simulator 2 (NS2) [1].

\subsection{Mobile Wimax (IEEE 802.16e)}

The mobile WiMAX (IEEE 802.16e) [2] is a mobile extension of the IEEE 802.16 standard [3].

IEEE 802.16 defines the specifications for radio metropolitan networks or WMAN (Wireless Metropolitan Area Network), offering broadband to achieve a high flow rate and using techniques to cover large areas [3].

The IEEE 802.16e is suitable for any kind of traffic thanks to its flexibility justified by its three MAC layers [2] and its use of IP protocol.

There are two kinds of Handover in the mobile WiMAX: Intra-ASN Handover (layer 2: no change of IP address) and Inter-ASN Handover (layer 3: IP address change) [4] [5]. For Intra-ASN, two mechanisms have been specified: Hard handover for the low speed and Soft handover for the high speed; and for Inter-ASN Handover it defines: Mobile IPv4 or ClientMIPv4, and Proxy-MIPv4 [4].

The architecture of 802.16e is composed of mobile stations (MS), that communicate freely (radio link) with base stations (BS), which act as an intermediates gateways with the terrestrial infrastructure of IP network. The base stations themselves are connected to the network elements called ASN-GW (gateways) which manages their connection with the IP network [4] [5]. 
The NAP (Network Access Provider) is an entity that provides the infrastructure for radio access to one or more providers of network services. It can control one or more ASN (Access Service Network) which is composed of one or more BS and one or more gateways.

The NSP (Network Service provider) is an entity that provides IP connectivity and network services to subscribers compatible with the level of service it establishes with subscribers. An NSP may also establish roaming agreements with other providers of network services and contractual agreements with third-party providers of application (for example, ASP: Application Service Provider) to provide IP services to subscribers.

A NSP control one or more CSN (Connectivity Service Network) which is the core of the WiMAX network.

The architecture of mobile WiMAX is presented in the figure 1:

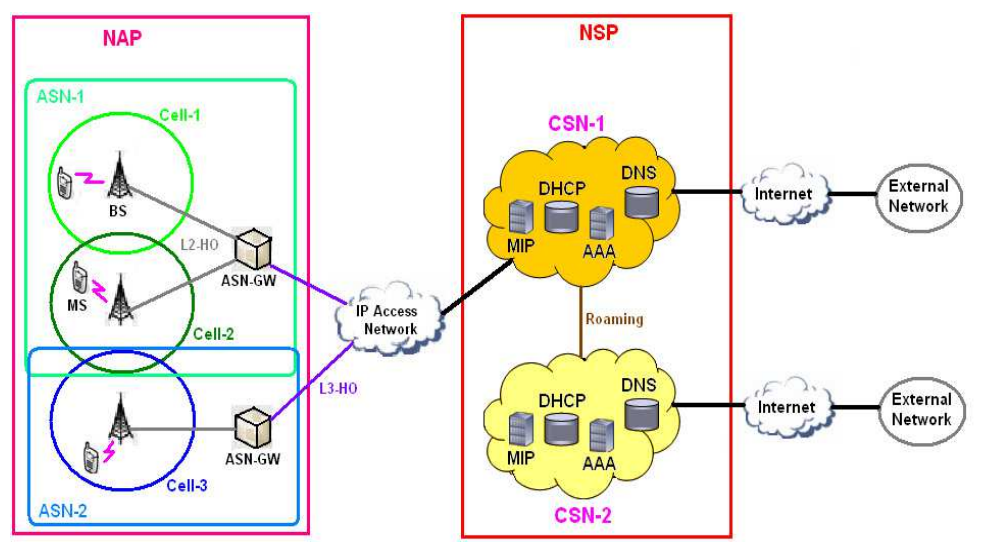

Fig. 1. IEEE 802.16e Architecture [4] [5]

\subsection{IEEE 802.11s}

IEEE 802.11s [6] [7] is an amendment being developed to the IEEE 802.11 WLAN standard, and aims to implement mobility on Ad-Hoc networks with acceptable debit.

In September 2003, IEEE formed the 802.11s SG which, in July 2004, became the "Extended Service Set (ESS) Mesh Networking" or 802.11s Task Group (TGs), and it is the most advanced group of the 802.11 WG.

The current objective of this TG is to apply mesh technology to WLANs by defining a Wireless Distribution System (WDS) used to build a wireless infrastructure with MAC-layer broadcast/multicast support in addition to the unicast transmissions. The TG should produce a protocol that specifies the installation, configuration, and operation of WLAN mesh. Moreover, the specification should include the extensions in topology formation to make the WLAN mesh self-configure and self-organized, and support for multi-channel, and multi-radio devices. At the MAC layer, a selection path protocol should be incorporated, instead of assigning the routing task to the network layer.

The WLAN Mesh architecture comprises the following IEEE 802.11 based elements: 
- Mesh Point (MP) which supports (fully or partially) mesh relay functions, and implement operations such as channel selection, neighbor discovery, and forming and association with neighbors. Additionally, MPs communicate with their neighbors and forward traffic on behalf of other MPs.

- Mesh Access Point (MAP= MP+AP) which is a MP but acts as an AP as well. Therefore, MAPs can operate in a WLAM Mesh or as part of legacy IEEE 802.11 modes.

- Mesh Portal (MPP=MP+Bridge) is another kind of MP that allows the interconnection of multiple WLAN meshes to form a network of mesh networks. Moreover, MPP can function as bridges or gateways to connect to other wired or wireless networks in the DS.

- $\quad$ Simple Station (STA): outside of the WLAN Mesh, connected via Mesh AP.

The architecture of IEEE 802.11 s is presented in the figure 2 below:

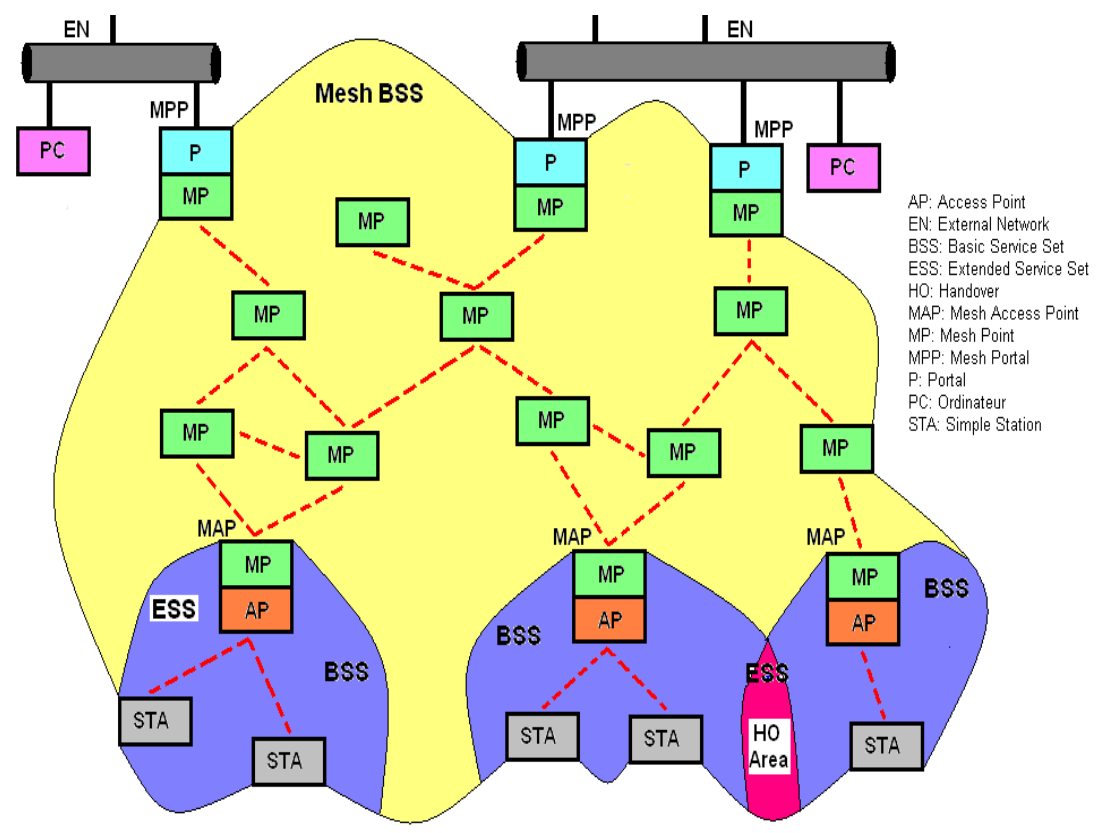

Fig. 2. IEEE 802.11s Architecture

\section{Vertical handover mechanisms proposed}

In our work, we analyze two vertical handover mechanisms that will be used between the mobile WiMAX and the IEEE 802.11s, and in this section we will present the two proposed mechanisms.

\subsection{MSCTP protocol}

The transport layer mobility is proposed as an alternative to the network layer mobility to support integrated mobility. The management of mobility in the transport layer is made exclusively by Stream Control Transmission Protocol (SCTP) [8] and its extension: Dynamic Address Reconfiguration (DAR) [9]. 
SCTP extended with DAR constitute Mobile SCTP (MSCTP) [10] [11] [12].

MSCTP was designed in order to avoid the connection disruptions observed with TCP or UDP during a change of IP address. It is a transport layer protocol similar to Transmission Control Protocol (TCP). It provides point-to-point communication oriented connection between applications running on different hosts. The major difference with TCP is the multihoming; it allows by multi-homing to manage multiple IP addresses in terminal nodes by conserving the point-to-point connection intact (see figure 3).
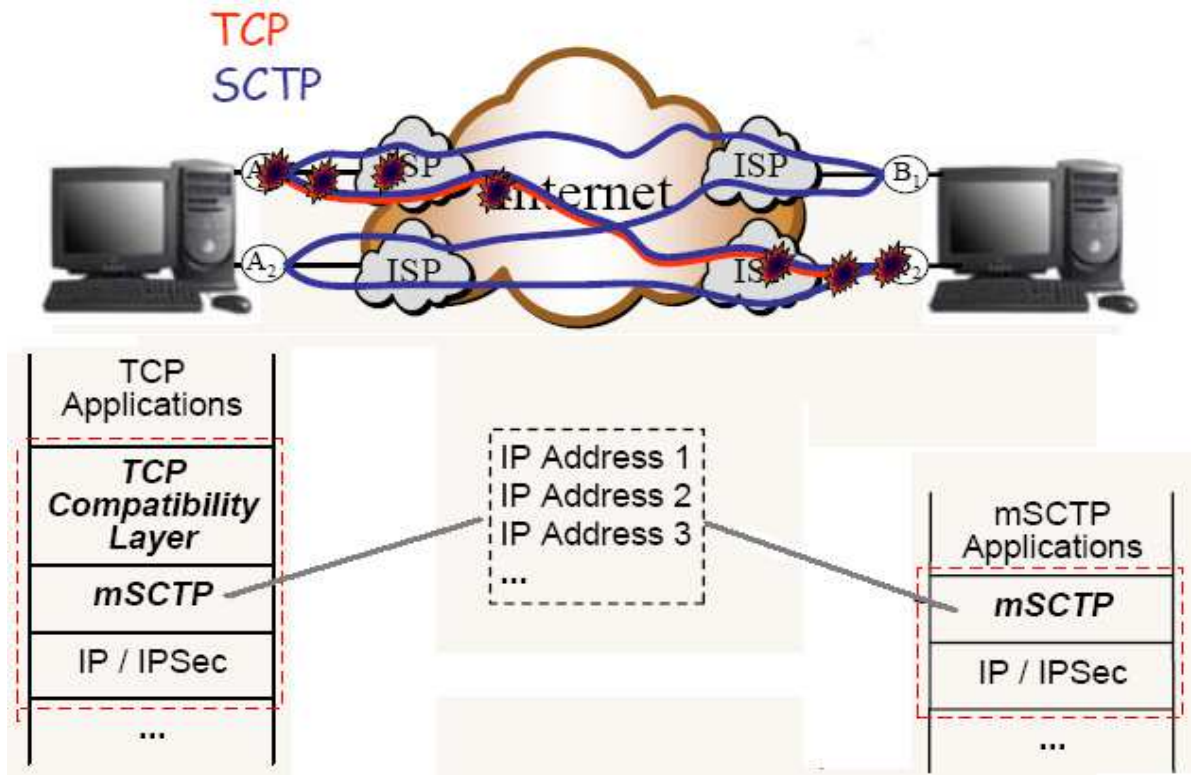

Fig. 3. MSCTP vs TCP and protocol stack

In the beginning of the communication between a mobile station (MS) and its correspondent $(\mathrm{CN})$ implementing both MSCTP protocol; in the MS, there is only one IP address chosen as primary address, and used as destination address for the current transmission. The other IP addresses are used only for retransmissions. The DAR extension allows to MS to add, delete and change IP addresses during a SCTP session, without affecting the connection established, by using address configuration messages.

During the communication with the $\mathrm{CN}$; when the MS changes from its home network to a foreign network passing by handover Area (at the beginning of the coverage area of foreign network), it receives an IP address from the foreign network either by contacting a DHCP, or by automatic configuration of IPv4 address. The MS is now able to establish other link with its $\mathrm{CN}$ through this second IP address obtained, and may become accessible via the foreign network. Then it sends its second IP address via the home network to its $\mathrm{CN}$, and the $\mathrm{CN}$ will add the new IP address to the association identifying the connection with the MS and sends an ACK to the MS to confirm. After, when the MS begin to leave the coverage area of its home network to the coverage area of the foreign network, it notifies the $\mathrm{CN}$ to assign the new IP address as primary IP address, which the CN approves with an ACK. 
The new primary IP address is now the second IP address obtained. The $\mathrm{CN}$ sends at this moment all the messages to the new IP address of MS via the foreign network. And finally, when the MS leave definitively the coverage area of the home network, it informs the CN to delete the first IP address of the association, which the CN confirms with an ACK [10] [11] [12]. To use MSCTP, the only requirement is that the both endpoints should implement MSCTP protocol.

By applying the MSCTP in the case of vertical handover between 802.16e and 802.11s, the protocol will use the multi-homing technique to open two IP sessions with the BS of the mobile WiMAX network and the AP of the Wireless mesh network to avoid the service interruption during handover.

\subsection{IEEE 802.21 or $\mathrm{MIH}$}

IEEE 802.21 or Media Independent Handover (MIH) [13] [14] [15] is a recent evolution for all networks, that providing capabilities to detect and initiate handover from one network to another. It designed a new function to control access to the lower layers (Layers 1 and 2). This new function provides new service access points (SAPs) and allows the information to be queried by the upper layers (Layer 3 and higher). Both mobile device and network hardware must implement the standard to work, but everything should remain backward compatible for non-MIH aware devices.

The standard allows simply to provide information that help to the initiation of handover, the selection of the network and the activation of the interface. The execution and the decision of handover is not part of the standard.

In MIH Function (MIHF), there are three services that allow the passage of messages along the stack. The table 1 below compiled from IEEE 802.21, outlines the basic functions of these services [14].

\begin{tabular}{|l|l|l|l|}
\hline $\begin{array}{l}\text { MIH } \\
\text { services }\end{array}$ & Origin & Destination & Use cases \\
\hline Event & $\begin{array}{l}\text { MIHF or lower } \\
\text { layer }\end{array}$ & $\begin{array}{l}\text { MIHF or upper layer } \\
\text { Remote or local stack }\end{array}$ & $\begin{array}{l}\text { Link up/down/going } \\
\text { down, transmissions } \\
\text { status }\end{array}$ \\
\hline Command & $\begin{array}{l}\text { MIHF or upper } \\
\text { layer }\end{array}$ & $\begin{array}{l}\text { MIHF or lower layer } \\
\text { Remote or local stack }\end{array}$ & switch links, get status \\
\hline Information & $\begin{array}{l}\text { Upper or lower } \\
\text { layer } \\
\text { Secure or insecure } \\
\text { port }\end{array}$ & $\begin{array}{l}\text { Upper or lower layer } \\
\text { Remote or local stack }\end{array}$ & $\begin{array}{l}\text { information elements } \\
\text { (IEs), neighbor reports }\end{array}$ \\
\hline
\end{tabular}

Table 1. MIH services

The MIH architecture is illustrated in the figure 4 below: 


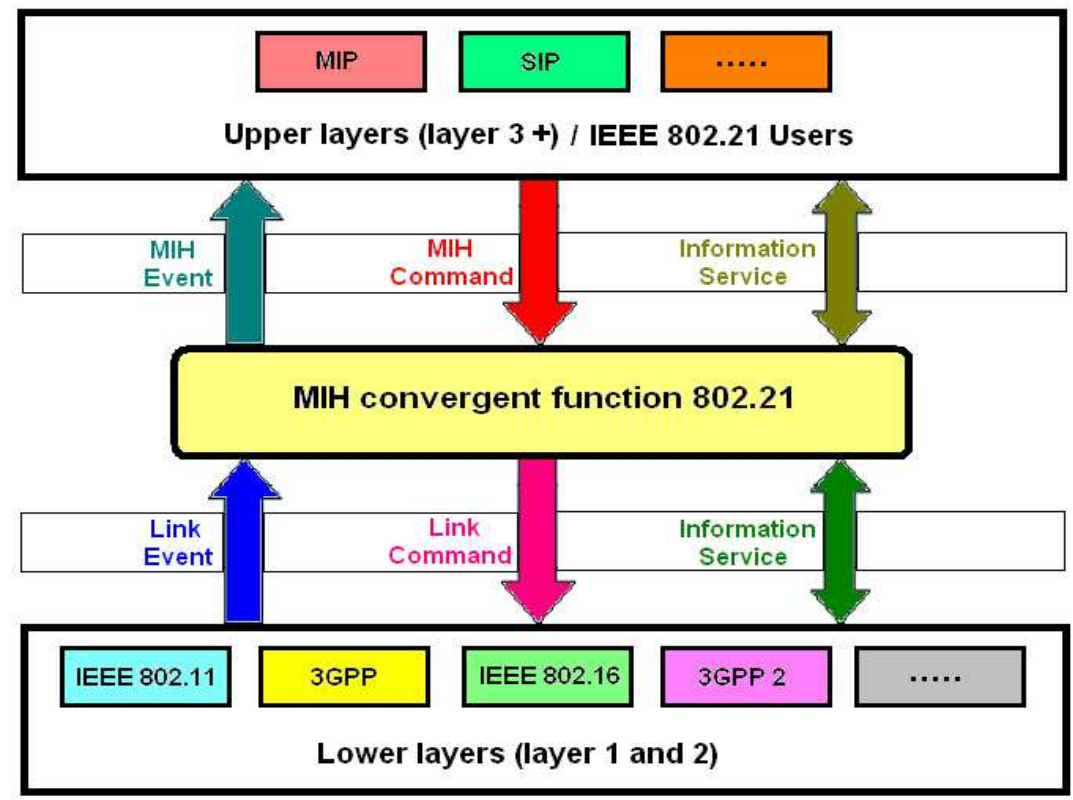

Fig. 4. MIH architecture

\section{Interconnection models proposed}

\subsection{Common simulation model and common scenario}

We describe in this section the common interconnection model proposed between IEEE 802.16e and IEEE 802.11s using MSCTP or IEEE 802.21 during the vertical handover; and the MS mobility scenarios between the two networks. We will assume an 802.16e cell with coverage of $1 \mathrm{~km}$ radius and an 802.11s cell with coverage of $300 \mathrm{~m}$ radius (these choices of radius values are based on the nature of the test environment that is an urban area which is not very dense). And, the two cells have a common area (handover area) with a variable and maximum distance of $180 \mathrm{~m}$ between both limits of cells (this choice of the surface of common area between cells is based on the time needed for the handover simulation).

The Base station (BS) of 802.16e network is linked to an ASN-GW that is linked too via IP network to a CSN (WiMAX ISP); the Access Point (AP) of 802.11s network is linked to a router that is linked too via IP network to a WIFI CSN; and the two CSNs are connected together and with the distant servers via Internet network.

We will evaluate the mechanisms through two mobility scenarios for the simulations: the case where the mobile move from 802.16e cell to 802.11s cell, and the opposite case. In the two scenarios, the mobile station (MS) traverses $200 \mathrm{~m}$ in $802.16 \mathrm{e}$ or $802.11 \mathrm{~s}$ cell, and traverses in handover area (common area between 802.16e and 802.11s cells) $100 \mathrm{~m}$.

We will propose three MS speeds to see the impact of speed increasing on the handover. So, we will propose: $5 \mathrm{~m} / \mathrm{s}=18 \mathrm{~km} / \mathrm{h} ; 10 \mathrm{~m} / \mathrm{s}=36 \mathrm{~km} / \mathrm{h}$; and $20 \mathrm{~m} / \mathrm{s}=72 \mathrm{~km} / \mathrm{h}$ as mobile speeds for the simulations. 


\subsection{Simulation model based on MSCTP protocol}

Based on common model proposed in the section before, with MSCTP protocol, the end users (the MS moving between the two cells and its correspondent node or server) must implement the MSCTP protocol.

The architecture of simulated model proposed for the interconnection between IEEE 802.16e and IEEE 802.11s using MSCTP is illustrated in the figure 5 below:

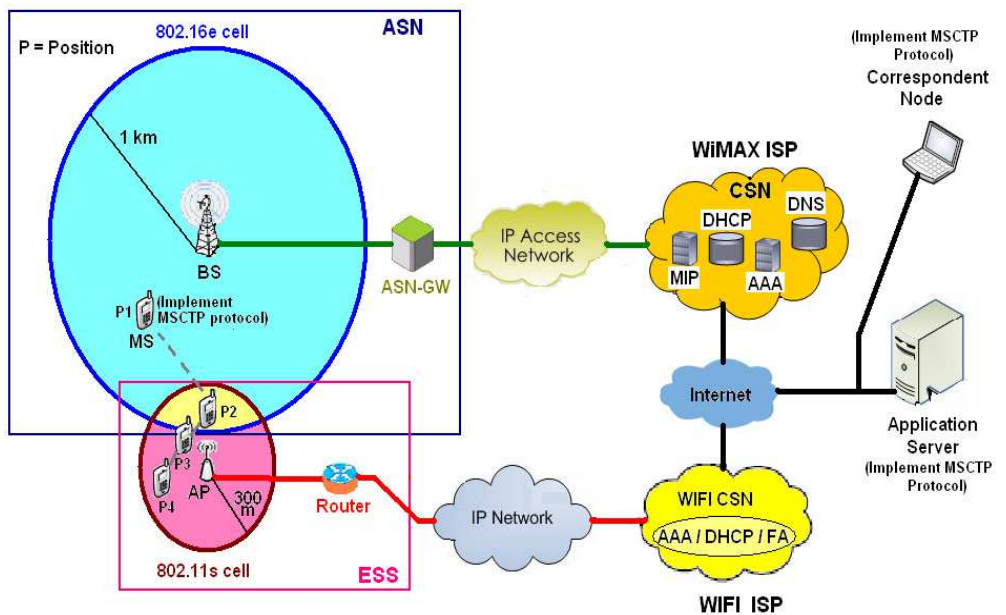

Fig. 5. Interconnection model using MSCTP

The exchange of information between the MS and its correspondent node $(\mathrm{CN})$ during the MS mobility scenario between IEEE 802.16e and IEEE 802.11s using MSCTP is illustrated in the figure 6 below:

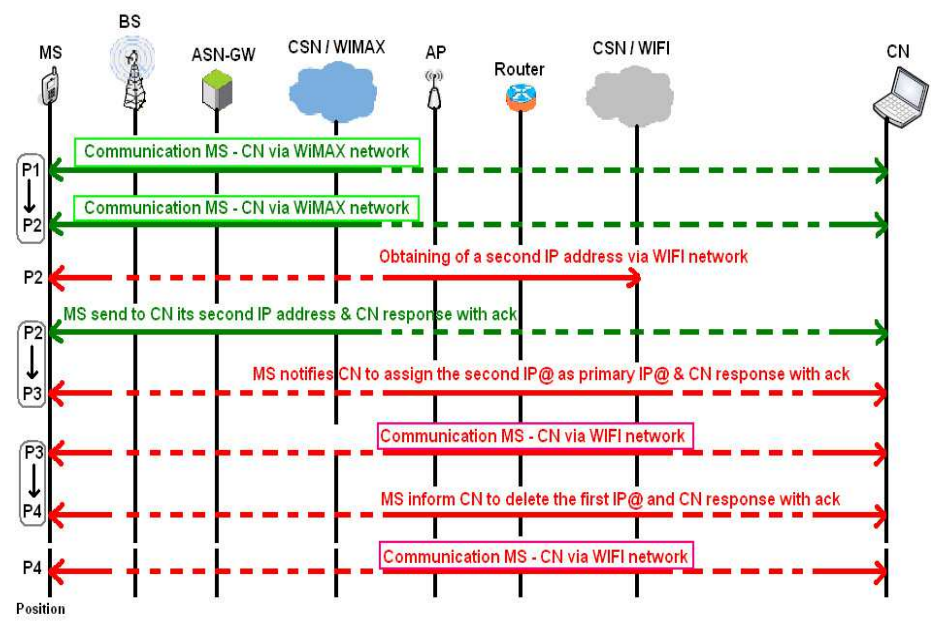

Fig. 6. Messages exchanged during simulation of the protocol MSCTP 


\subsection{Simulation model based on $\mathbf{8 0 2 . 2 1}$ architecture}

Based on the common model already described; with IEEE 802.21 architecture, an MIH server must be implemented in the Internet network, more precisely between the WiMAX CSN, and the WIFI CSN; and MIH modules must be implemented in the two CSNs and in the MS.

The architecture of the interconnection model proposed between the two networks using IEEE 802.21 is illustrated in the figure 7 below:

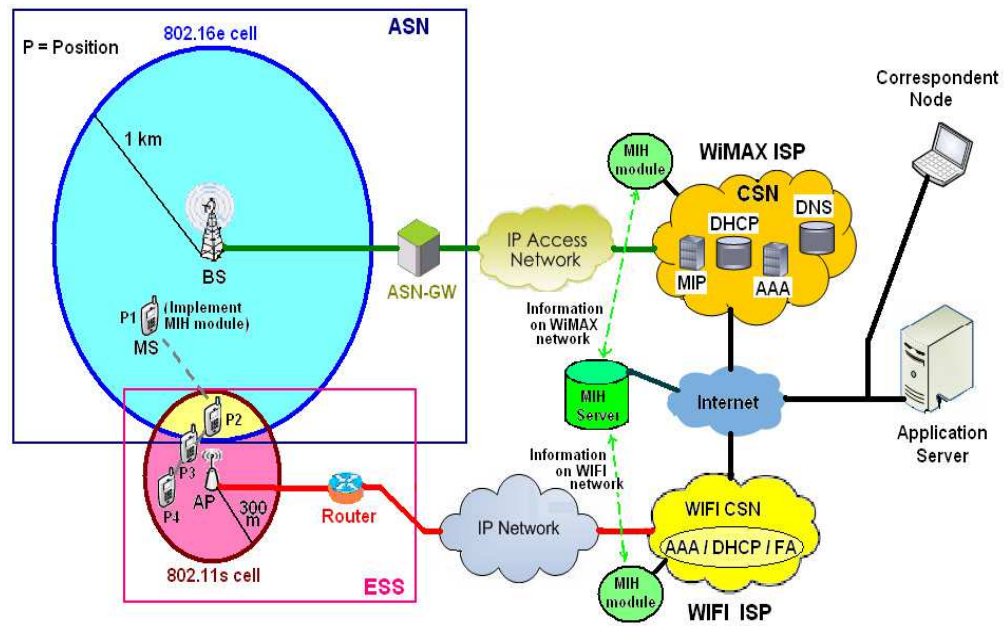

Fig. 7. Interconnection model using IEEE 802.21

The exchange of information between the MS and its correspondent node $(\mathrm{CN})$ during the MS mobility scenario between IEEE 802.16e and IEEE 802.11s using the MIH module is illustrated in the figure 8 below:

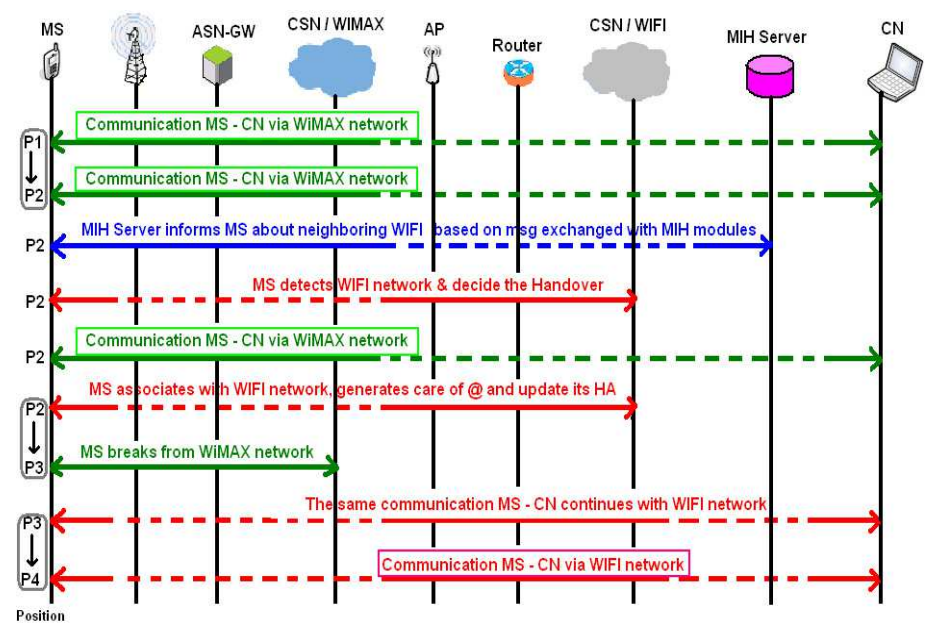

Fig. 8. Messages exchanged during simulation of the protocol MIH 
For the simulations, we choose to use Proxy Mobile IP (PMIP) [16] as layer 3 protocol that interacts with lower layers via MIH module.

PMIP is an amelioration of MIP, it introduces a functional entity called Proxy Mobile IP to help MIP traversal across VPN or "NAT and VPN" gateways. The PMIP is in the path between MS and its corresponding HA (Home Agent), and acts as a surrogate MS and HA.

PMIP does not involve a change in the point of attachment address when the user moves, and there is no need for the terminal to implement a client MIP stack.

\subsection{Simulation parameters under NS2 simulator}

During the simulations and with the two handover mechanisms, we will test three traffics types: the VoIP with a fixed size to 160 byte and a rate of 300 packet/sec, the Data with a fixed size to 640 bytes and a rate of $200 \mathrm{packet} / \mathrm{sec}$, and the video streaming with a fixed size to 1280 bytes and a rate of 100 packet/sec (optimal values usually chosen in NS2).

Under NS2, with MSCTP protocol we use the CBR over MSCTP traffic type; and with IEEE 802.21 architecture, we use the CBR over UDP traffic type.

The duration of one simulation are fixed to 250 seconds, and the results are calculate every 10 seconds in 802.16 e or 802.11 s cell area, and every 5 seconds during the handover process in common area.

For the two networks: IEEE 802.16s and IEEE 802.11s, the simulation parameters under NS2 are illustrated in the table 2 below:

\begin{tabular}{|l|l|l|}
\hline & IEEE 802.16e & IEEE 802.11s \\
\hline Transmission Power (Pt_) & $15 \mathrm{~W}$ & $0.2818 \mathrm{~W}$ \\
\hline Receiving Threshold (RXThresh_) & $7.59375 \mathrm{e}-11 \mathrm{~W}$ & $1.76148 \mathrm{e}-10 \mathrm{~W}$ \\
\hline $\begin{array}{l}\text { Carrier Sending Threshold } \\
\text { (CSThresh_) }\end{array}$ & $4.34219 \mathrm{e}-12 \mathrm{~W}$ & $3.32874 \mathrm{e}-11 \mathrm{~W}$ \\
\hline Coverage Radius (Distance D) & $1 \mathrm{~km}$ & $300 \mathrm{~m}$ \\
\hline Radio Propagation Model & $\begin{array}{l}\text { Two-Ray Ground [17] } \\
P_{r}(d)=\frac{P_{t} G_{t} G_{r} h_{t}{ }^{2} h_{r}{ }^{2}}{d^{4} L}\end{array}$ \\
\hline Transmit Antenna Gain (Gt_) & $1 \mathrm{~dB}$ & \\
\hline Receive Antenna Gain (Gr_) & $1 \mathrm{~dB}$ & \\
\hline System Loss (L_) & $1 \mathrm{~dB}$ & \\
\hline Transmit Antenna Height (ht_) & $1.5 \mathrm{~m}$ & OFDM \\
\hline Receive Antenna Height (hr_) & $1.5 \mathrm{~m}$ & $2.4 \mathrm{Ghz}$ \\
\hline Modulation & OFDMA & \multicolumn{2}{|l|}{} \\
\hline Frequency (Freq_) & $3.5 \mathrm{Ghz}$ &
\end{tabular}

Table 2. Simulation parameters 


\subsection{Performance criteria}

The performance criteria adopted in our simulations to compare MSCTP and IEEE 802.21 mechanisms in the case of vertical handover between $802.11 \mathrm{~s}$ and 802.16e networks are: End-to-end delay, packets loss ratio and debit. These parameters are the main criteria of QoS measuring in the networks. To evaluate the QoS degree of these criteria, we will compare simulation results obtained with theoretical thresholds estimated to evaluate the QoS depending on traffic type.

\section{Results}

\subsection{End-to-end delays}

The end-to-end delay is a very important parameter to evaluate the QoS for the real time traffic. It is the time needed for a packet to be transmitted across a network from source to destination.

In this section, we will calculate the delays of packets during the simulation time for the three mobile speeds and the three traffic types; with the two vertical handover techniques: $\mathrm{MIH}$ architecture and MSCTP protocol; and applying the two scenarios: handover from 802.16 e to 802.11 s and from 802.11 s to $802.16 \mathrm{e}$.

We start by present the results for the VoIP traffic (see figures 9 and 10).

In the two figures 9 and 10, first for all the curves we see that during the handover process, the delays obtained with MSCTP are slightly lower than those obtained with the MIH; and the handover are executed with MIH before that with MSCTP.

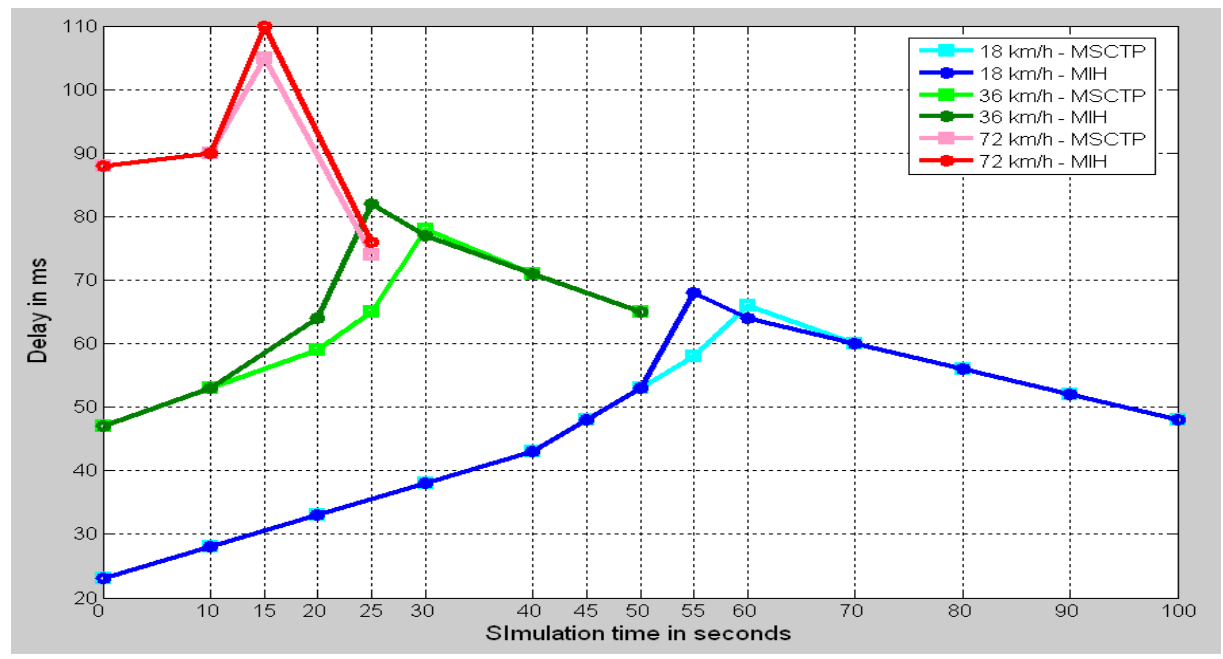

Fig. 9. Delay of HO from 802.11 s to 802.16 e / VoIP 


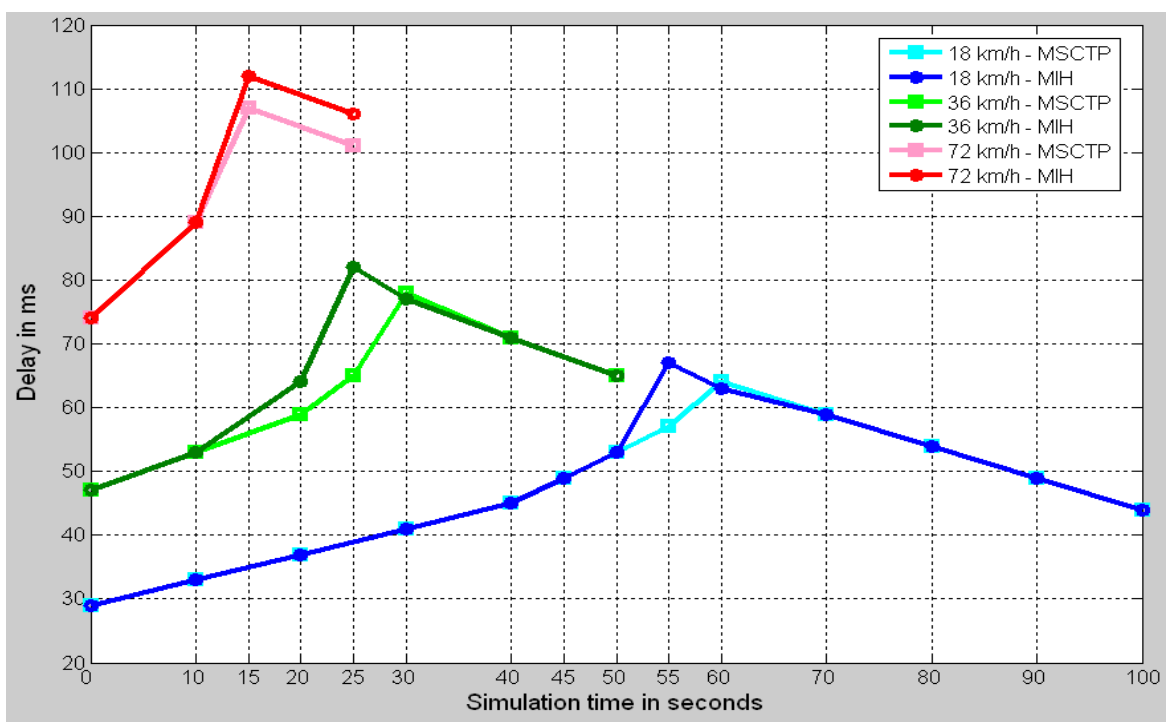

Fig. 10. Delay of HO from 802.16e to 802.11s / VoIP

With the two speeds: 18 and $36 \mathrm{~km} / \mathrm{h}$, the QoS level is accepted for the VoIP traffic that needed a minimum delay of $100 \mathrm{~ms}$ [18]; but with the speed equal to $72 \mathrm{~km} / \mathrm{h}$, the QoS degrades during the handover.

Finally, with a speed of $18 \mathrm{~km} / \mathrm{h}$, the QoS is better in 802.11s network; with $36 \mathrm{~km} / \mathrm{h}$ the QoS is equivalent in the two cells; and with $72 \mathrm{~km} / \mathrm{h}$ of speed the QoS is better in 802.16e cell.

We present now the results of data traffic (see figures 11 and 12):

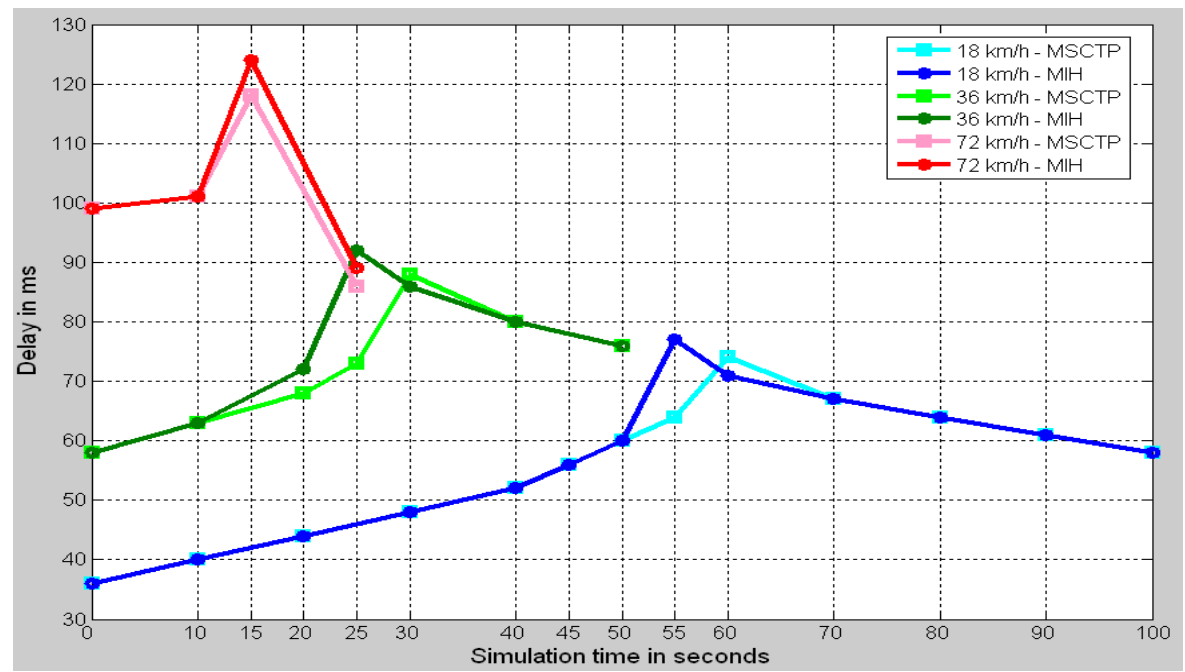

Fig. 11. Delay of HO from 802.11s to 802.16e / Data 


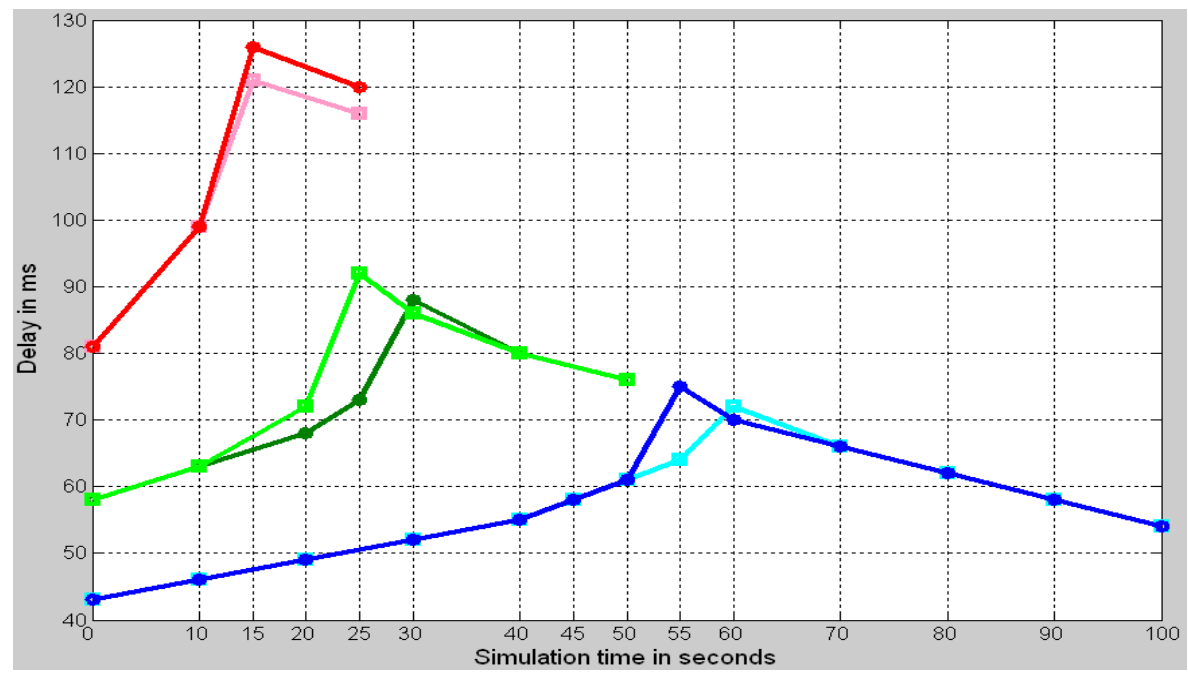

Fig. 12. Delay of HO from 802.16 e to 802.11 s / Data

With the data traffic, there is no delay constraint, so we can consider the QoS level acceptable for all the cases.

We note that the delays obtained with data are higher than those obtained with the VoIP traffic; for example with MSCTP protocol and with a speed of $18 \mathrm{~km} / \mathrm{h}$, the maximum delay obtained of the VoIP traffic is $65 \mathrm{~ms}$ versus $73 \mathrm{~ms}$ of the data traffic.

We present finally in this section the results of video streaming traffic (see figures 13 \& 14):

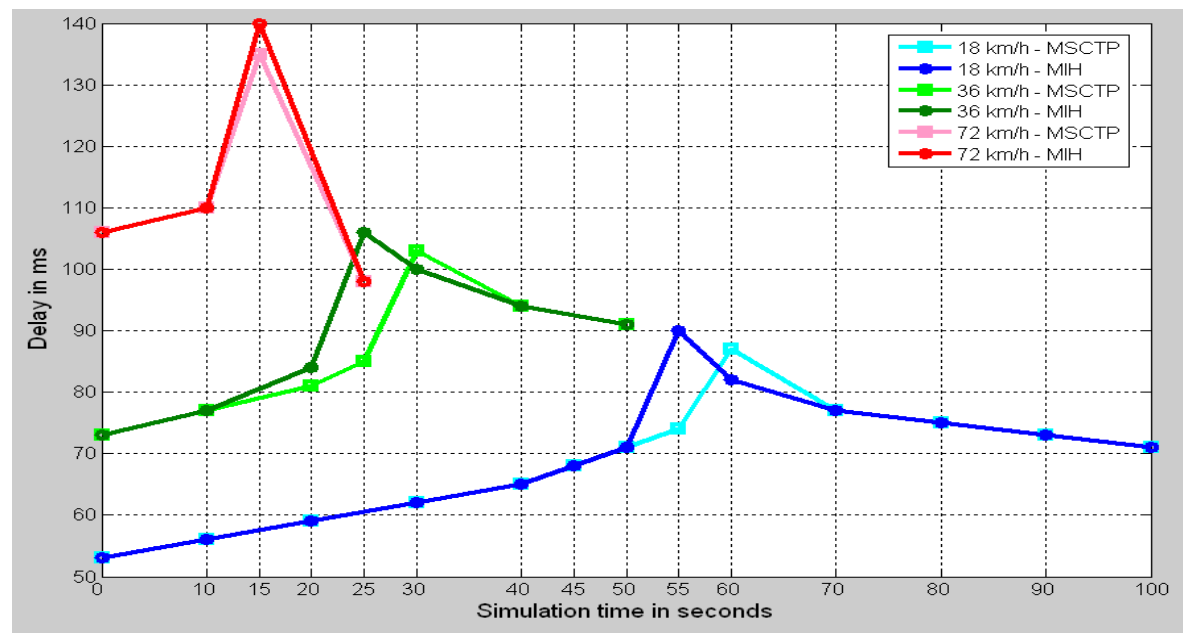

Fig. 13. Delay of HO from 802.11s to 802.16e / Video 


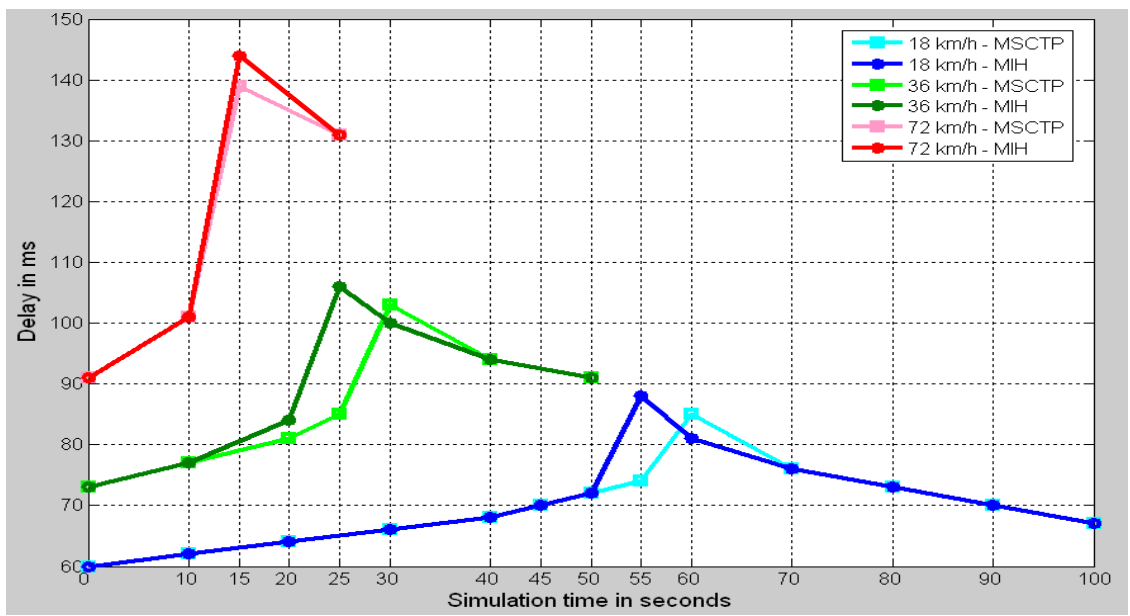

Fig. 14. Delay of HO from 802.16e to 802.11 s / Video

With the video traffic, the delay values increase comparing by the VoIP or the data traffic.

With the speed of $18 \mathrm{~km} / \mathrm{h}$, the delays not exceed $100 \mathrm{~ms}$; with the speed of $36 \mathrm{~km} / \mathrm{h}$ the delays exceed slightly $100 \mathrm{~ms}$ during the handover; and with the speed of $72 \mathrm{~km} / \mathrm{h}$, the delay values exceed largely $100 \mathrm{~ms}$ during the handover and in the 802.11 s cell.

The delays with MSCTP are slightly lower than those with MIH.

\subsection{Packet loss ratio}

We calculate in this section the percentage of lost packets with the same cases as those described in the section 4.1. We start with the VoIP traffic (see figures 15 and 16):

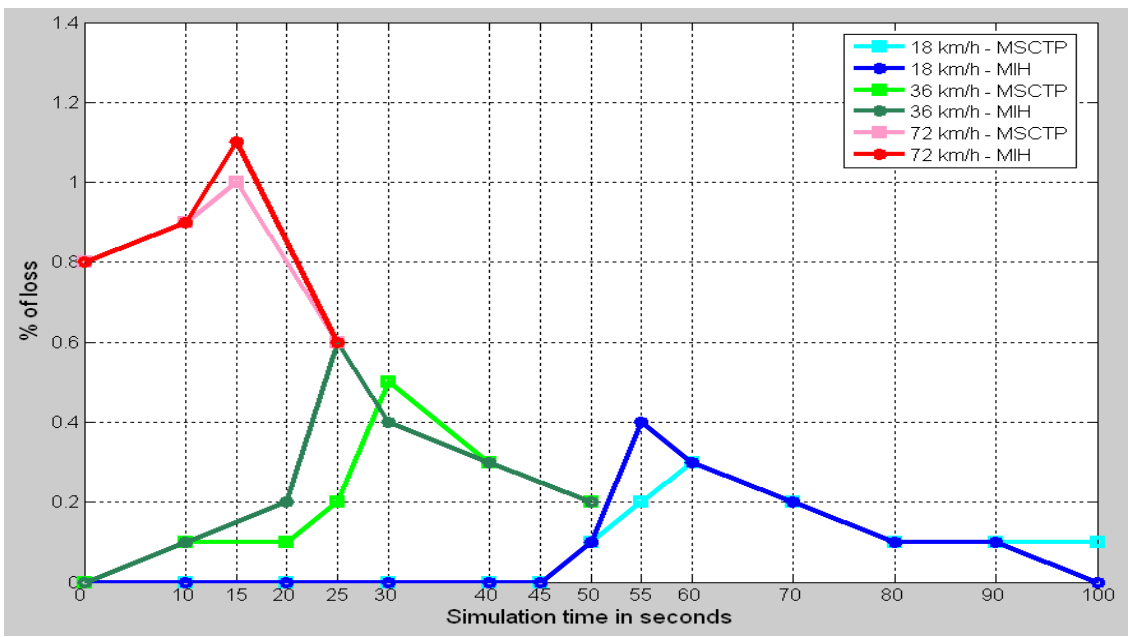

Fig. 15. Percentage of packets lost with HO from 802.11 s to $802.16 \mathrm{e} /$ VoIP 


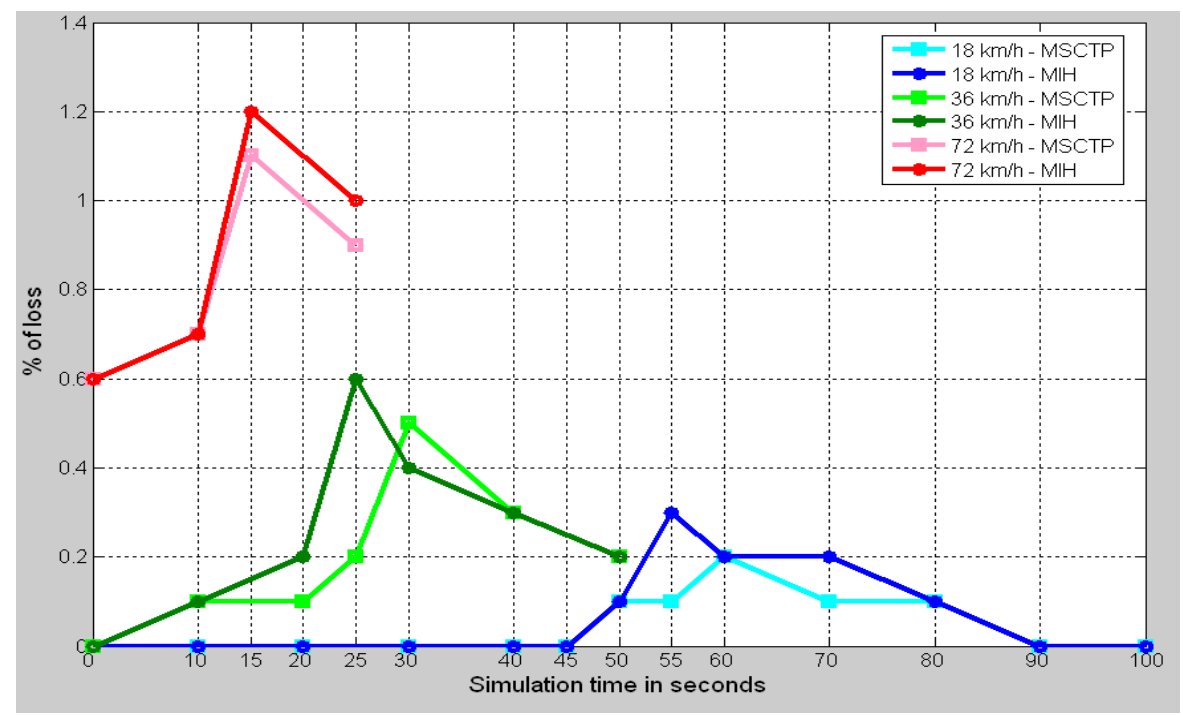

Fig. 16. Percentage of packets lost with HO from 802.16e to 802.11s / VoIP

During the Handover, the only results that converge to the threshold of $1 \%$ [18] required by the VoIP traffic] are those corresponding to the speed of $72 \mathrm{~km} / \mathrm{h}$. For the two other speeds the QoS level is acceptable.

We present now the results of data traffic (see figures 17 and 18):

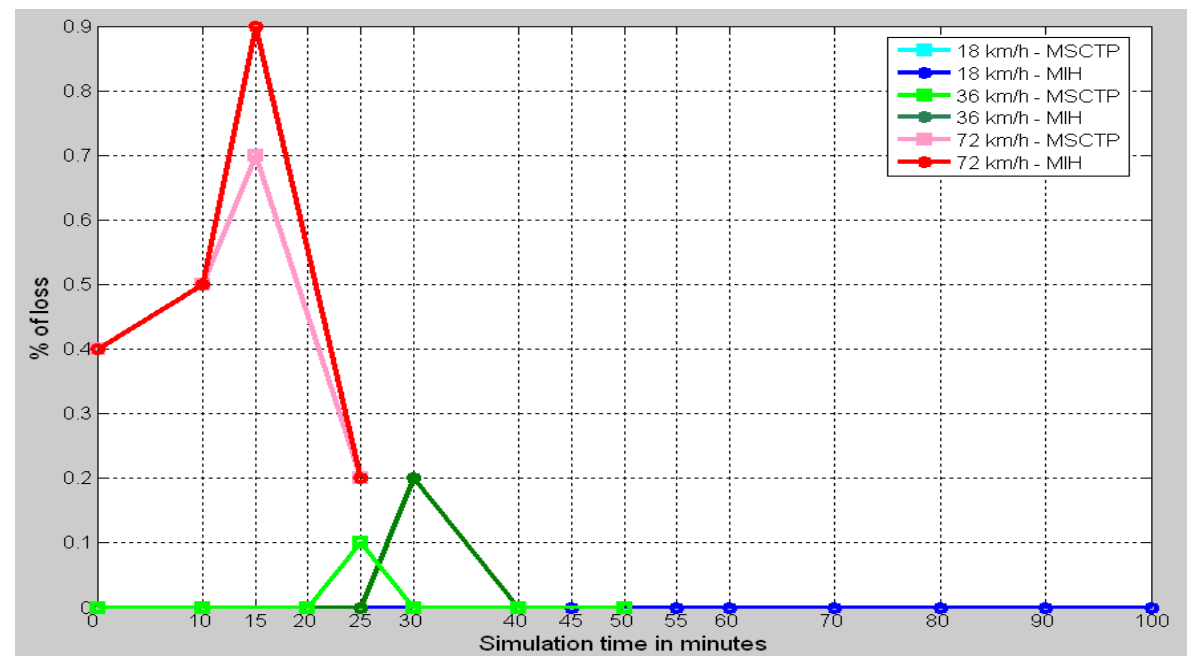

Fig. 17. Percentage of packets lost with HO from 802.11s to 802.11e / Data 


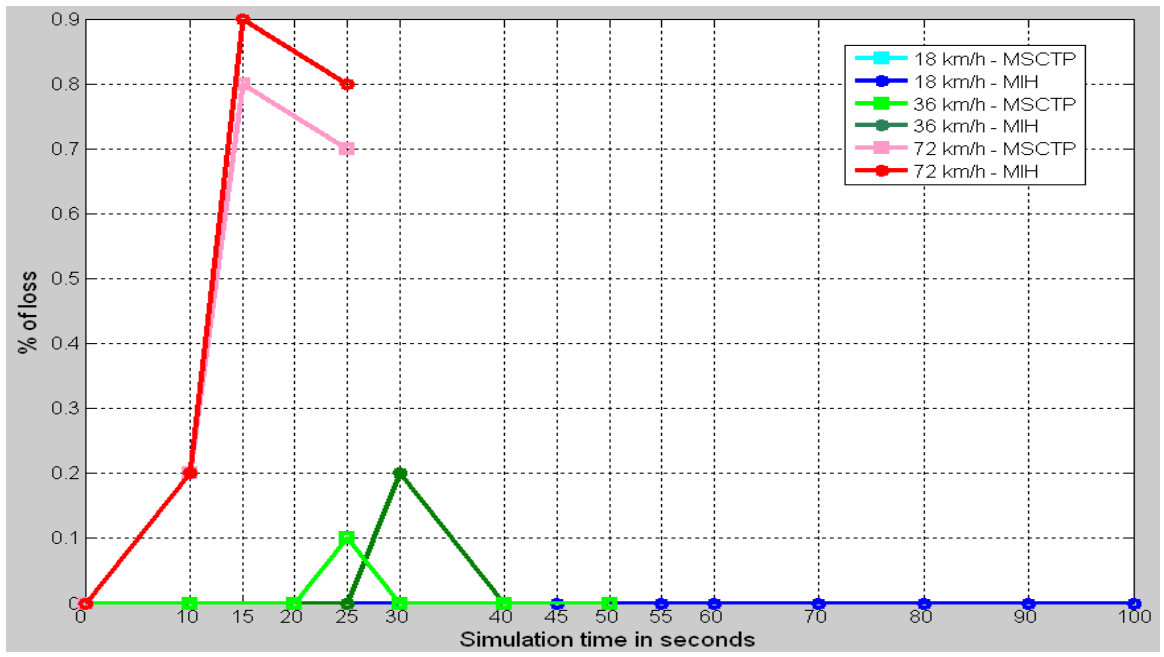

Fig. 18. Percentage of packets lost with HO from 802.16e to 802.11s / Data

With the data traffic, the percentage of loss is zero with a speed of $18 \mathrm{~km} / \mathrm{h}$, and it is acceptable with a speed of $36 \mathrm{~km} / \mathrm{h}$. It has a maximum of $0.1 \%$ with MSCTP and $0.2 \%$ with $\mathrm{MIH}$ during the Handover.

But with a speed of $72 \mathrm{~km} / \mathrm{h}$, the percentage of loss is not acceptable during the handover and in the 802.11s cell.

We pass now to video streaming traffic (see figures 19 and 20):

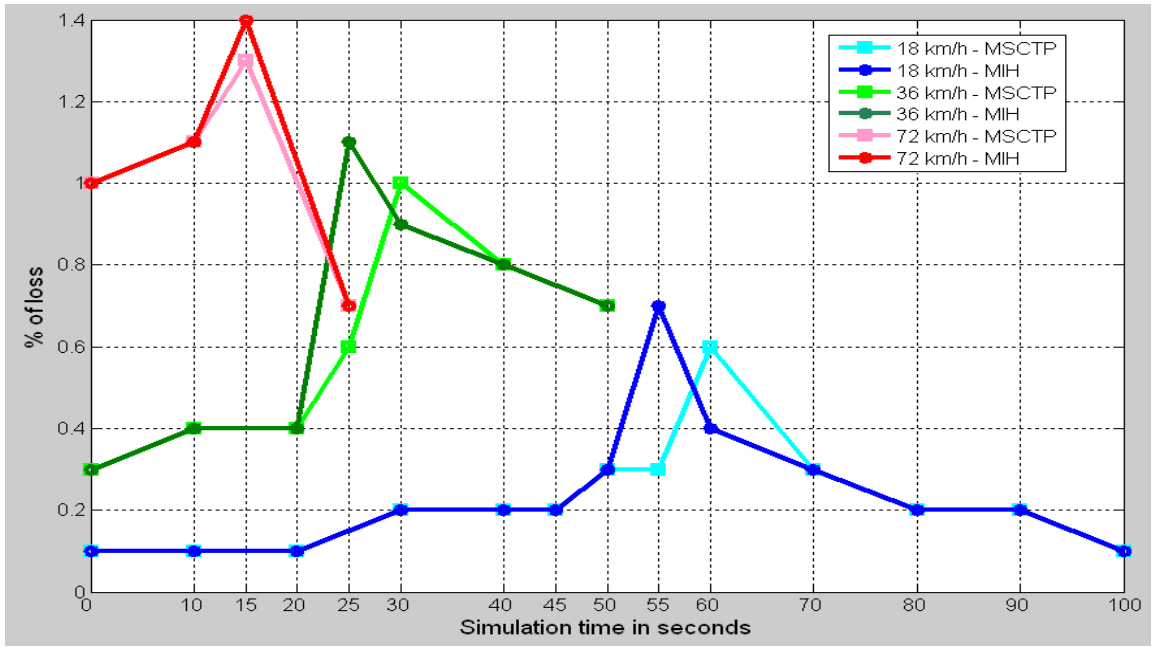

Fig. 19. Percentage of packets lost with HO from 802.11s to 802.16e / Video 


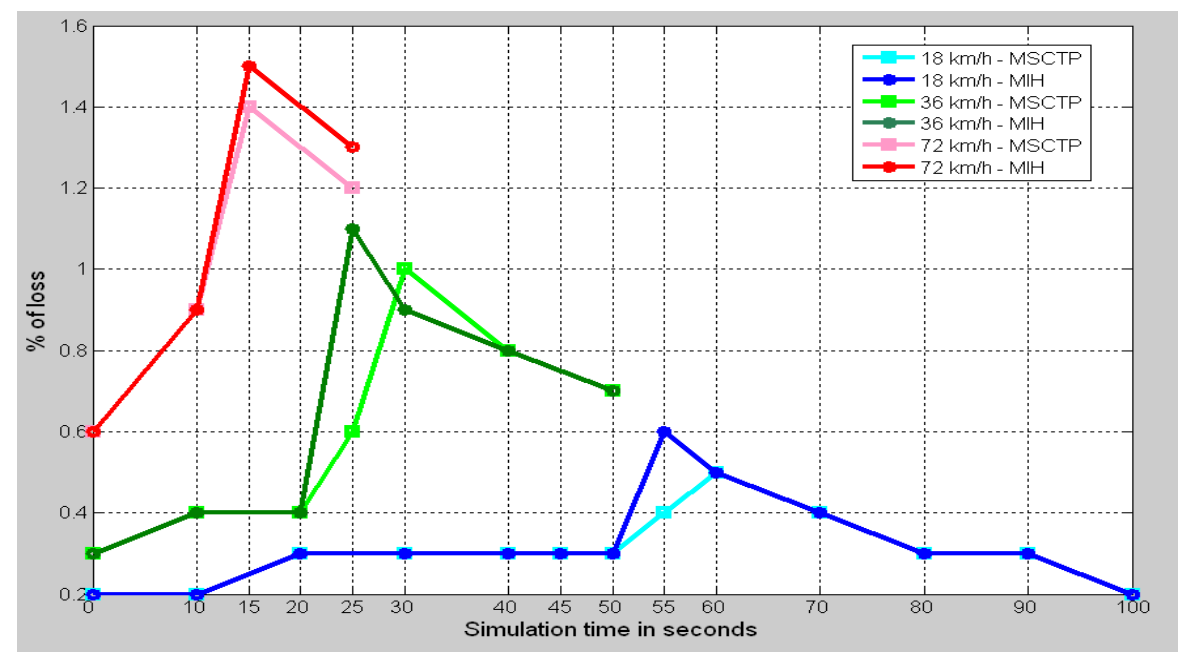

Fig. 20. Percentage of packets lost with HO from 802.16e to 802.11s / Video

With the video traffic, the loss values are higher than those with VoIP or data traffic, and with a speed of $18 \mathrm{~km} / \mathrm{h}$ the results are acceptable because they not exceed $1 \%$ which is a maximum of loss required for video traffic [18].

We note that the case of handover from 802.16 e to 802.11 s produce results slightly better than those obtained with the opposite case during the handover.

With the speed of $36 \mathrm{~km} / \mathrm{h}$, the maximum of the \% of loss exceeds slightly $1 \%$ with $\mathrm{MIH}$, and is equal to $1 \%$ with MSCTP; and the results are equivalent in the two ways of handover.

With a speed of $72 \mathrm{~km} / \mathrm{h}$, the results are not acceptable with the two mechanisms during the handover because they exceed largely $1 \%$, and the results of the handover from 802.11 s to 802.16e are better than those of the opposite case. And finally with this speed, the results are acceptable in the 802.16 e cell but not in the 802.11 s cell.

\subsection{Debit}

Finally, in this section we will evaluate the debit experimented by the mobile during the handover cases already proposed for the simulations in section 4.1. We start with the VoIP traffic (see figures 21 and 22).

Concerning the debit of VoIP traffic and with a minimum required by this type of traffic fixed to $4 \mathrm{~kb} / \mathrm{s} \mathrm{[18];} \mathrm{the} \mathrm{all} \mathrm{cases} \mathrm{proposed} \mathrm{with} \mathrm{the} \mathrm{two} \mathrm{speeds:} 18$ and $36 \mathrm{~km} / \mathrm{h}$ present an acceptable debit; but with the speed of $72 \mathrm{~km} / \mathrm{h}$ and during the handover, the results are lower than $4 \mathrm{~kb} / \mathrm{s}$, and the debit obtained with MSCTP during the handover is slightly higher than that obtained with MIH. 


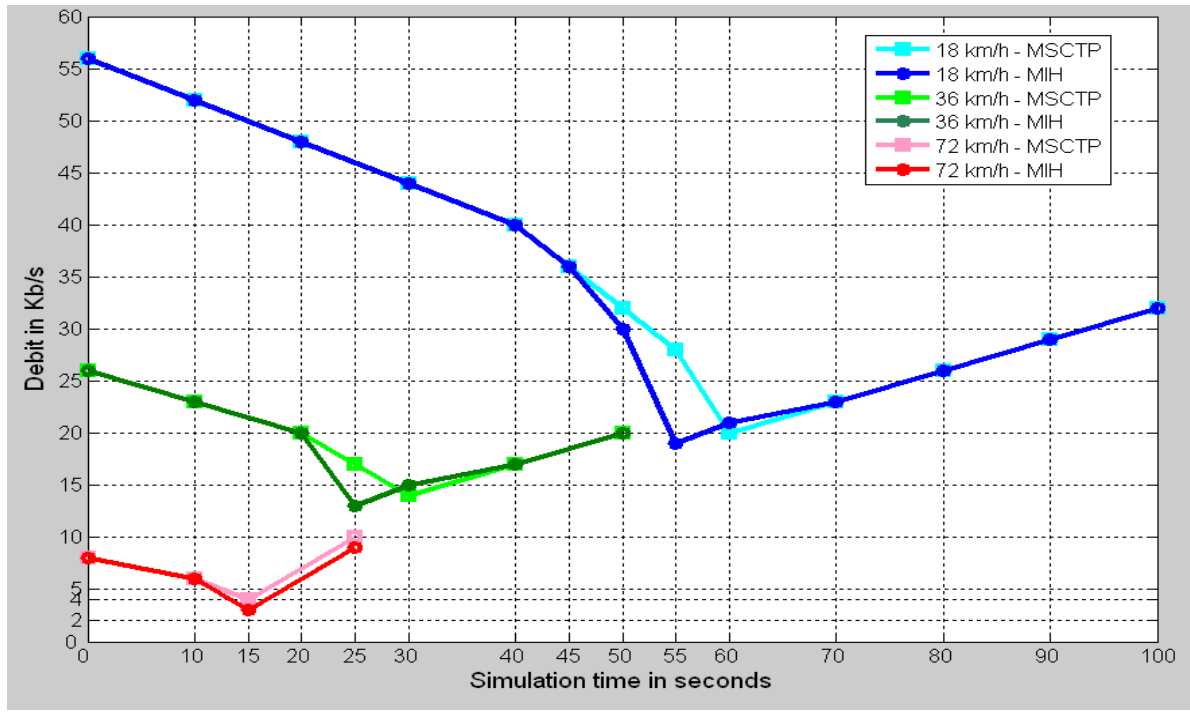

Fig. 21. Debit of HO from 802.11s to 802.16e / VoIP

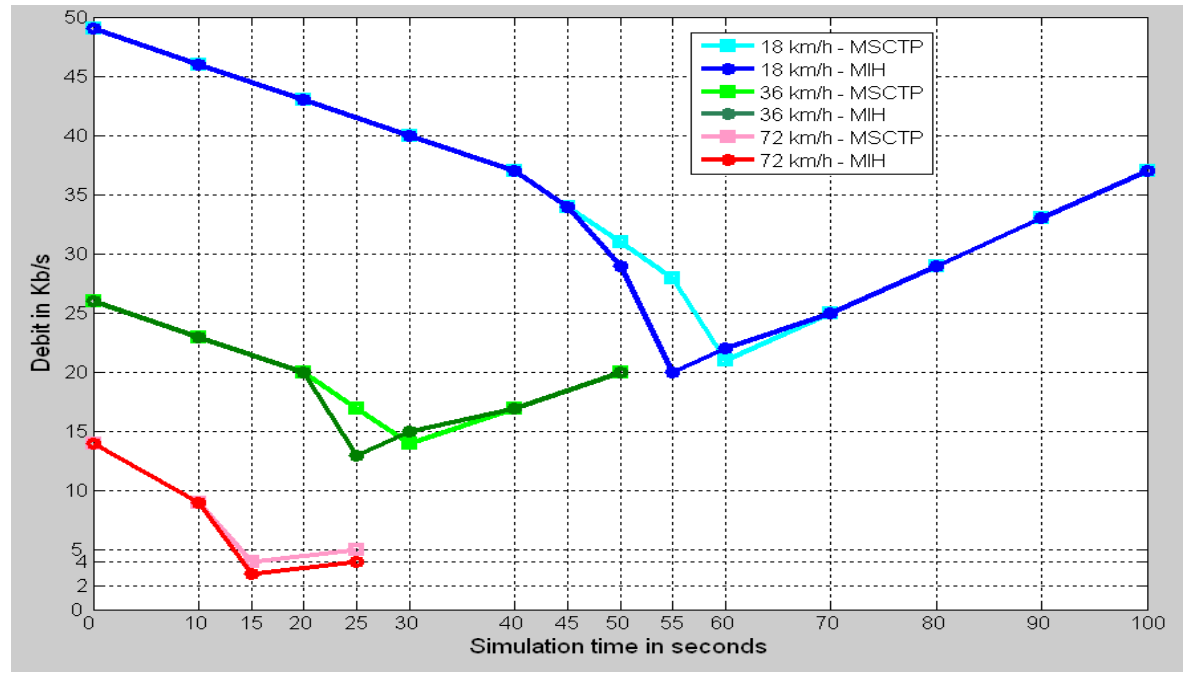

Fig. 22. Debit of HO from 802.16e to 802.11 s / VoIP

We present now the results of data traffic (see figures 23 and 24): 


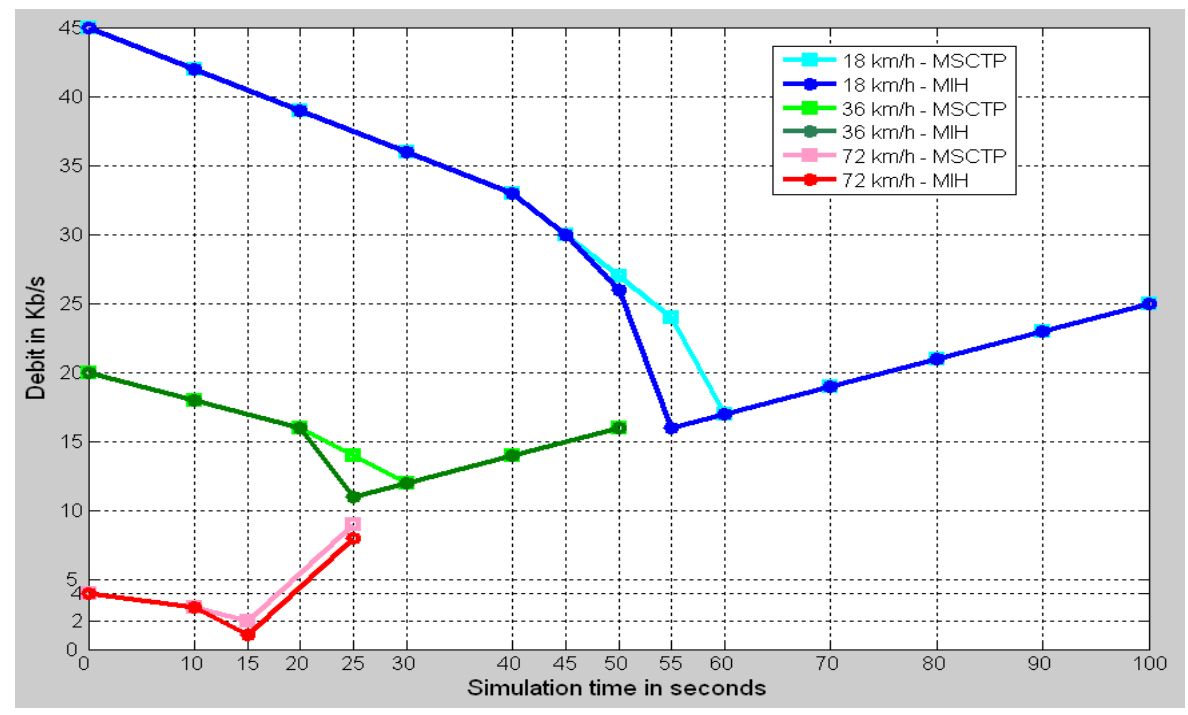

Fig. 23. Debit of HO from 802.11s to 802.16e / Data

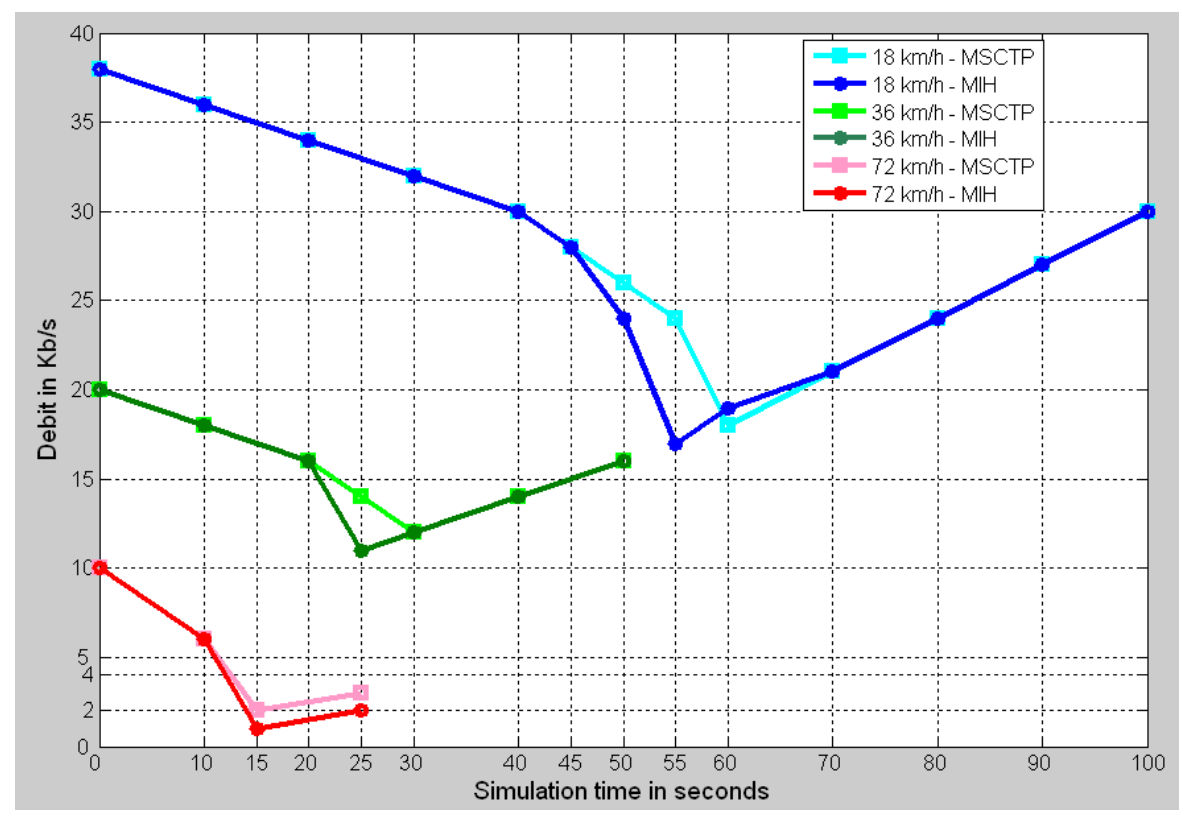

Fig. 24. Debit of HO from 802.16e to 802.11s / Data 
With the data traffic, the debit decrease when the speed increase. The debit is better in 802.11s cell when the speed is weak, and it is better in 802.16e when the speed is high.

We present finally the results of video traffic (see figures 25 and 26):

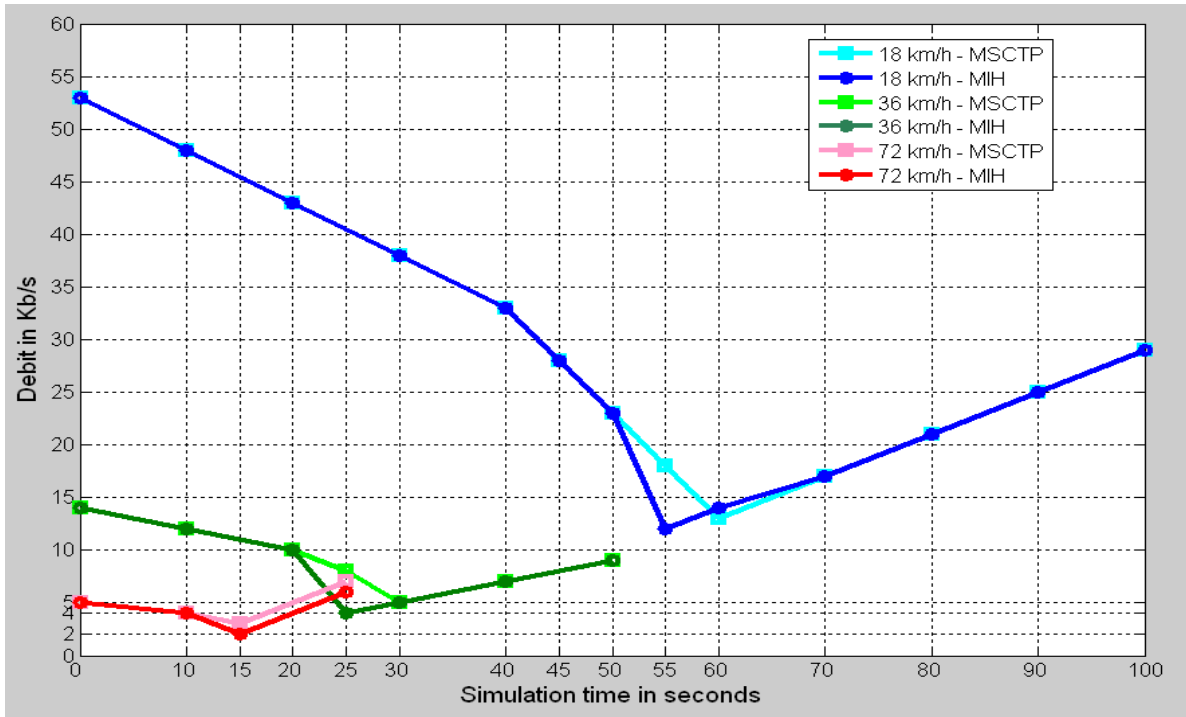

Fig. 25. Debit of HO from 802.11s to 802.16e / Video 


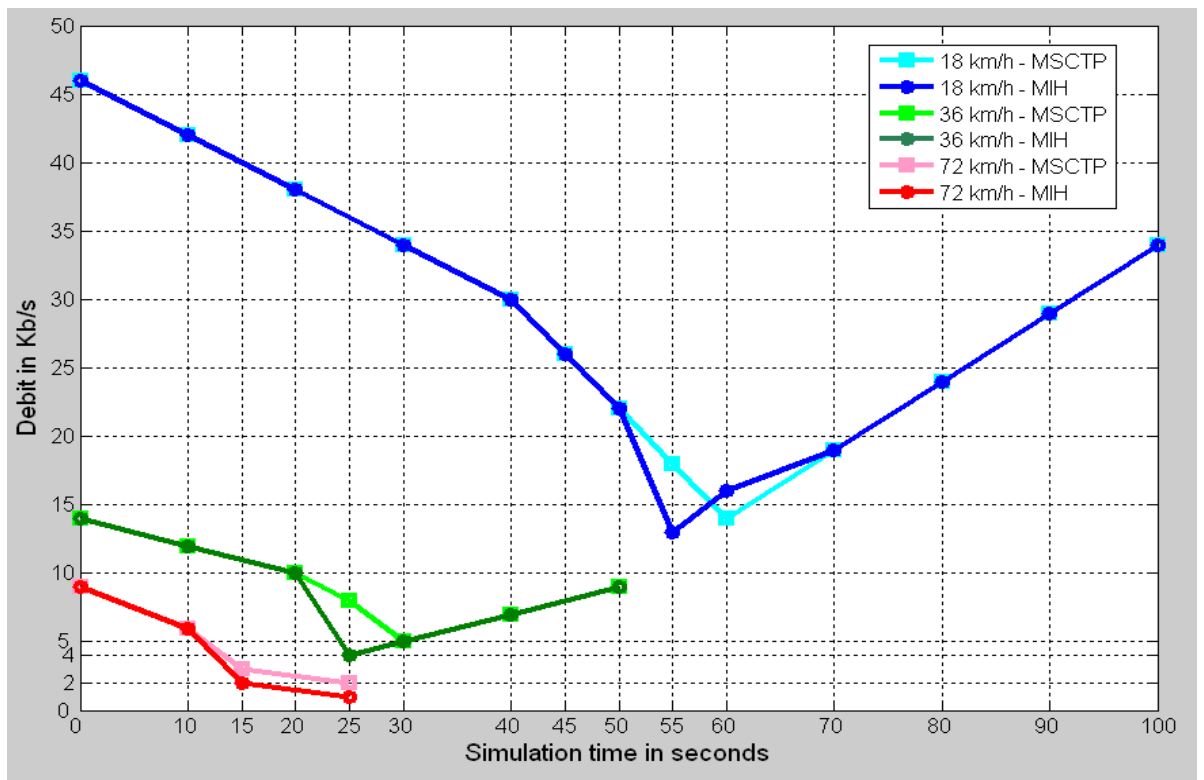

Fig. 26. Debit of HO from 802.16e to 802.11s / Video

With the video traffic, the debit values decrease comparing to the other traffic types.

\section{Conclusion}

The interoperability and the vertical handover between different networks present currently a real challenge to overcome. The difference of networks operation is the main reason of this problem. And, for pass to the $4 \mathrm{G}$ networks, it is important to resolve this problem of interoperability between different networks.

Our work has focused on the interconnection between two wireless radio networks of the IEEE 802 family, and we are concentrating on the QoS aspect for several traffics types especially during the handover process. For doing that, we have proposed two interconnection models based on two recent handover mechanisms, and we have simulated those two models with three mobile speeds and in the both directions of networks.

Observing the results obtained, we can conclude that with a low or medium speed of displacement of a mobile station, the both techniques: IEEE 802.21 and MSCTP present a good solution during the vertical handover. With the two techniques, there are very few interruptions during the vertical handover. But based on details of simulation results, we notice that with MSCTP protocol we obtained a QoS level slightly better than that obtained with MIH architecture. 
Also the handover from 802.11s to 802.16e generates results better than the opposite case of handover. But with a high speed, it is the opposite rather because the mobile WIMAX supports better the increasing speeds; and also the results in this case are still not acceptable comparing by QoS level needed for each traffic type.

It should be noted that during all the simulations, the scenarios proposed does not include cell congestion or lack of available resources.

For future work, we will propose interconnection models between networks of different family, we will mix a network world with a telecommunication world, and we will try to propose a handover mechanism adapted to the two entities that we will define.

\section{References}

[1] The Information Science Institute (ISI), "The Network Simulator-NS-2", http://www.isi.edu/ nsnam/ns/.

[2] EEE Std, "Air Interface for Fixed and Mobile Broadband Wireless Access Systems," IEEE 802.16e, Part 16, February 2006.

[3] IEEE Std, "Air Interface for Fixed Broadband Wireless Access Systems," Local and metropolitan area networks, Part 16, 2004.

[4] arviz Yegani, "WiMAX Overview," White paper, IETF-64 Cisco Systems, 2005.

[5] WiMAX Forum, "WiMAX End-to-End Network Systems Architecture," Draft Stage 2: Architecture Tenets, Reference Model and Reference Points, June 2007.

[6] Steven Conner, Jan Kruys, Kyeongsoo Kim and Juan Carlos Zuniga, "IEEE 802.11s Tutorial," Overview of the Amendment for Wireless Local Area Mesh Networking, IEEE 802 Planary, November 2006.

[7] Guido R. Hiertz, Sebastian Max, Rui Zhao, Dee Denteneer and Lars Berlemann, "Principles of IEEE 802.11s," Computer Communications and Networks, 2007, ICCCN 2007.

[8] RFC 2960, “Stream Control Transmission Protocol," IETF, 2000.

[9] Stewart R., \& al., IETF, "Stream Control Transmission Protocol (SCTP) Dynamic Address Reconfiguration," IETF Internet, Draft, draft-ietf-tsvwg-addip-sctp-13.txt, November 2005.

[10] Koh, S., \& al., "mSCTP for Soft Handover in Transport Layer," IEEE Communication Letters, Vol. 8, No.3, pp.189-191, March 2004.

[11] Memory graduation, Esteban Zimanyi, "Performance analysis of vertical Handover between UMTS and 802.11 networks," 2005.

[12] Deng Feng, "Seamless Handover between CDMA2000 and 802.11 WLAN using mSCTP," Thesis, 2006.

[13] IEEE 802.21 tutorial, July 2006.

[14] Jared Stein, “Survey of IEEE 802.21 Media Independent Handover Services," April 2006.

[15] V. Gupta, "IEEE 802.21 standard and metropolitan area networks: Media Independent Handover services", Draft P802.21/D05.00, April 2007.

[16] K. Leung, G. Dommety, P. Yegani \& K. Chowdhury, "Mobility Management Using Proxy Mobile IPv4", Internet Draft, IETF, 2007. 
[17] Information Sciences Institute (ISI), “NSNAM web pages, 18.2 Two-Ray Ground reflection model," http://www.isi.edu/nsnam/ns/doc/node218.html, January 2009.

[18] WiMAX Community, “WiMAX fundamentals, 1.7.3 Quality of Service”, June 2007. 


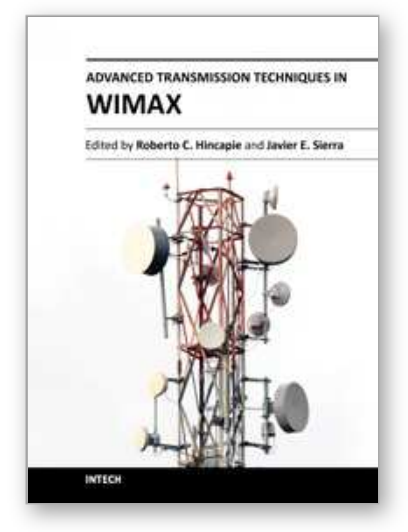

\author{
Advanced Transmission Techniques in WiMAX \\ Edited by Dr. Roberto Hincapie
}

ISBN 978-953-307-965-3

Hard cover, 336 pages

Publisher InTech

Published online 18, January, 2012

Published in print edition January, 2012

This book has been prepared to present the state of the art on WiMAX Technology. The focus of the book is the physical layer, and it collects the contributions of many important researchers around the world. So many different works on WiMAX show the great worldwide importance of WiMAX as a wireless broadband access technology. This book is intended for readers interested in the transmission process under WiMAX. All chapters include both theoretical and technical information, which provides an in-depth review of the most recent advances in the field, for engineers and researchers, and other readers interested in WiMAX.

\title{
How to reference
}

In order to correctly reference this scholarly work, feel free to copy and paste the following:

Tarek Bchini and Mina Ouabiba (2012). Interaction and Interconnection Between 802.16e \& 802.11s, Advanced Transmission Techniques in WiMAX, Dr. Roberto Hincapie (Ed.), ISBN: 978-953-307-965-3, InTech, Available from: http://www.intechopen.com/books/advanced-transmission-techniques-in-wimax/interactionand-interconnection-between-802-16e-802-11s

\section{INTECH}

open science | open minds

\section{InTech Europe}

University Campus STeP Ri

Slavka Krautzeka 83/A

51000 Rijeka, Croatia

Phone: +385 (51) 770447

Fax: +385 (51) 686166

www.intechopen.com

\section{InTech China}

Unit 405, Office Block, Hotel Equatorial Shanghai

No.65, Yan An Road (West), Shanghai, 200040, China

中国上海市延安西路65号上海国际贵都大饭店办公楼 405 单元

Phone: +86-21-62489820

Fax: +86-21-62489821 
(C) 2012 The Author(s). Licensee IntechOpen. This is an open access article distributed under the terms of the Creative Commons Attribution 3.0 License, which permits unrestricted use, distribution, and reproduction in any medium, provided the original work is properly cited. 